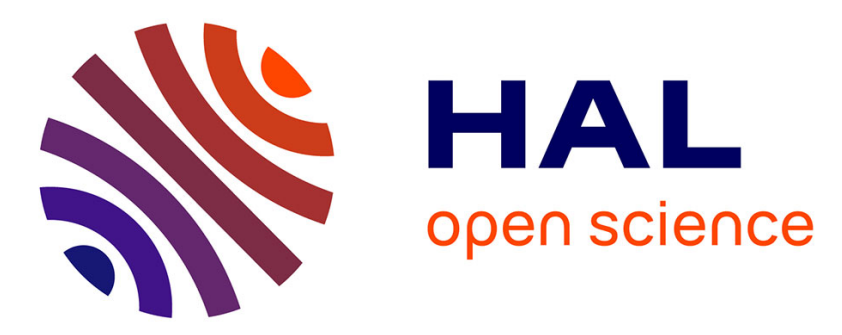

\title{
Les préoccupations et la tonalité émotionnelle des enseignants débutants lors de leurs premières expériences en classe \\ Luc Ria, Marc Durand
}

\section{- To cite this version:}

Luc Ria, Marc Durand. Les préoccupations et la tonalité émotionnelle des enseignants débutants lors de leurs premières expériences en classe. Les Dossiers des sciences de l'éducation , 2001, 5, pp.111-123. hal-00803986

\section{HAL Id: hal-00803986 https://hal.science/hal-00803986}

Submitted on 24 Mar 2013

HAL is a multi-disciplinary open access archive for the deposit and dissemination of scientific research documents, whether they are published or not. The documents may come from teaching and research institutions in France or abroad, or from public or private research centers.
L'archive ouverte pluridisciplinaire HAL, est destinée au dépôt et à la diffusion de documents scientifiques de niveau recherche, publiés ou non, émanant des établissements d'enseignement et de recherche français ou étrangers, des laboratoires publics ou privés. 


\title{
Les préoccupations et la tonalité émotionnelle des enseignants débutants lors de leurs premières expériences en classe
}

\author{
Luc Ria \\ Laboratoire d'Anthropologie des Pratiques Corporelles \\ Clermont Ferrand
}

Marc Durand

Équipe ESTEREL, Institut Universitaire de Formation des Maîtres, Montpellier

\section{Numéro thématique : Les pratiques enseignantes : contributions plurielles.}

Les Dossiers des Sciences de l'Education, 5, 111-123.

\section{Résumé:}

Cette recherche décrit et interprète l'expérience d'une enseignante débutante en Éducation Physique lors de sa première année d'enseignement. La méthode pour décrire et interpréter son expérience accorde le primat à ses émotions durant son expérience, à ses préoccupations face aux élèves, et à la manière de concrétiser ses préoccupations dans l'action. Les résultats montrent combien la tonalité émotionnelle et les préoccupations de l'enseignante influencent son action lors de situations d'enseignement ordinaires. Ils montrent la nécessité de dévoiler la dimension subjective de l'expérience professionnelle des enseignants débutants en formation initiale.

\footnotetext{
Abstract:

This study describes and interprets the beginning teacher's experience in Physical Education during her first teaching year. The method to describe and interpret her experience is based on a primacy allowed to her emotions during her experience, to her preoccupations in front of their students, and to the way she copes with her preoccupations into action. The results indicate how teacher's emotional tonality and preoccupations format her action during ordinary teaching situations. They show the usefulness of revealing the subjective dimension of beginning teacher's professional experience in teacher education programs.
}

\section{Mots clefs:}

Enseignant débutant / Expérience professionnelle / Préoccupations / Emotions. 
Notre recherche décrit et interprète l'expérience d'enseignants débutants en Éducation Physique lors de leur première année professionnelle et plus particulièrement la tonalité émotionnelle de leur expérience en classe. Elle s'inscrit dans un programme de recherche dont l'objet plus large est de dévoiler la composante subjective du travail humain.

\section{La théorie sémiologique du cours d'expérience}

Notre recherche adopte la théorie sémiologique du cours d'expérience ${ }^{1}$ reposant sur deux présupposés fondamentaux. Premièrement, l'activité est située, c'est-à-dire qu'elle est indissociable de la situation dans laquelle elle prend forme et par conséquent, elle doit être étudiée in situ. Deuxièmement, le couplage entre l'acteur et son monde se transforme en permanence au cours de l'activité et des interactions.

La théorie sémiologique du cours d'expérience s'attache au niveau de l'expérience qui est significatif pour l'acteur, c'est-à-dire montrable, racontable et commentable par lui à tout instant de son déroulement. La définition de cet objet théorique est fondée sur le postulat que le niveau de l'activité qui est montrable, racontable et commentable par l'acteur est un niveau d'organisation relativement autonome par rapport à d'autres niveaux d'analyse de l'activité et qu'il peut donner lieu à des observations, descriptions et explications valides et utiles.

Le signe hexadique

La théorie sémiologique étudie l'expérience humaine à partir de signes hexadiques ${ }^{2}$ reliant entre elles six composantes dont chacune est une catégorie particulière de l'expérience de l'acteur. Ces six composantes du signe hexadique $^{3}$ sont à la fois indissociables et distinctes les unes des autres. Nous les présentons ici:

L'engagement (E) dans la situation est constitué par le faisceau de préoccupations ou d'intérêts immanents de l'acteur découlant de ses actions passées. Il est l'ouverture pour l'acteur d'un champ de possibles susceptibles de se réaliser.

L'actualité potentielle (A) correspond aux attentes structurées de l'acteur relatives à sa situation à un instant donné. Elles spécifient l'état attendu de son couplage à la situation lors de la concrétisation de ses préoccupations. l'acteur.

Le référentiel $(\mathrm{S})$ correspond à l'ensemble des connaissances issues des cours d'expérience passés de

L'engagement (E), l'actualité potentielle (A) et le référentiel (R) constituent la structure d'attente de l'acteur (E-A-S) correspondant à une disposition à agir émergeant dans la situation de façon globale et diffuse.

Le représentamen $(\mathrm{R})$ correspond à ce qui, à un instant donné, fait effectivement signe pour l'acteur, c'est-àdire à ce qui fait "effectivement choc" pour l'acteur compte tenu de son engagement (E), de ses attentes (A) et de ses connaissances $(S)$. Le représentamen $(R)$ opère une transformation de la structure d'attente (E-A-S) en (eR-aR-sR). Ce processus d'actualisation en (eR-aR-sR) constitue la portion de (E-A-S) sélectionnée par (R). Ainsi, des préoccupations $(\mathrm{eR})$, des attentes $(\mathrm{aR})$ et des connaissances $(\mathrm{sR})$ émergent chez l'acteur en fonction du représentamen (R). Par exemple, la préoccupation (eR) "Contrôler un élève" est focalisée parmi l'ensemble des préoccupations de l'enseignant au moment où ce dernier perçoit les pitreries d'un élève (les pitreries lui font signe). Cette préoccupation (eR) émerge en fonction des connaissances $(s R)$ qu'il mobilise à cet instant au sujet de l'élève: "Cet élève perturbe régulièrement la classe" et par rapport à la façon d'intervenir qu'il estime efficace en fonction de son expérience: "L'intervention auprès de cet élève doit servir d'exemple pour la classe". La préoccupation (eR) se prolonge par des attentes concrètes (aR) dans la situation de classe liées à "l'arrêt immédiat des pitreries de l'élève" et "à la démonstration publique du contrôle de la situation face aux élèves".

L'unité élémentaire $(\mathrm{U})$ est la fraction significative de l'activité pour l'acteur à un instant t. Elle est l'expression synthétique de son activité.

L'interprétant (I) correspond à la construction de nouvelles connaissances ou à la confirmation (ou l'infirmation) d'une connaissance déjà acquise.

Les émotions dans la théorie du cours d'expérience

\footnotetext{
${ }^{1}$ Theureau $(1992,2000)$ a développé, à partir de la phanéroscopie de Peirce (1978), une théorie sémiologique du cours d'expérience s'inscrivant dans le cadre d'une "anthropologie cognitive située". Ces formalisations théoriques ont donné lieu à de nombreuses recherches empiriques notamment dans le domaine de l'enseignement (Durand, 1998, 2000; Ria, 2001; Ria et al., 2001).

${ }^{2}$ Ces signes hexadiques, dans le prolongement théorique du signe triadique de Peirce (1978), se rattachent à l'hypothèse de la "pensée signe" selon laquelle l'homme pense et agit par signes. Décrire et interpréter le cours d'expérience implique d'identifier les processus de construction de ces signes en action (de cette pensée en action) qui sont des unités de signification.

${ }^{3}$ Le lecteur intéressé par une présentation plus complète de la théorie du cours d'expérience peut se référer à Theureau $(1992,2000)$, et pour des prolongements empiriques concernant l'expérience professionnelle d'enseignants débutants à Ria (2001).
} 
Dans la théorie sémiologique du cours d'expérience, les émotions ne constituent pas une catégorie supplémentaire de l'expérience humaine. Elles sont immanentes à l'action et l'essence même de l'expérience. Elles "infiltrent" et "colorent" les différentes catégories de l'expérience de l'acteur (Ria, 2001).

Pour la documentation des émotions au niveau du cours d'expérience, nous distinguons: (a) des états affectifs relatifs au flux continu des émotions d'arrière-plan (Damasio, 1999). Ces états affectifs syncrétiques correspondent à la révélation immédiate et diffuse, à la fois indubitable et inanalysable, de soi et de sa situation. Ils sont "montrés" globalement par l'acteur à l'aide d'une échelle ordinale de "Confort - Inconfort", (b) des sentiments relatifs à la conscientisation ponctuelle et partielle de l'expérience émotionnelle. Ils constituent ce que l'acteur peut raconter, commenter, mettre en mots lors du dévoilement de son expérience. Ils émergent sur la base des états affectifs de l'acteur, (c) des émotions-types relatives à la typification des émotions vécues par l'acteur. Ces émotionstypes traduisent la conscientisation par l'acteur de la régularité de certaines de ses expériences émotionnelles dans des situations relativement similaires.

Participants

\section{Méthode}

Les participants à ce programme de recherche étaient huit enseignants stagiaires au cours de leur année préprofessionnelle de PLC2 (Professeurs des Lycées et Collèges - $2^{\text {ème }}$ année). Ils étaient âgés de 23 à 25 ans et avaient obtenu le concours du CAPEPS (Certificat d'Aptitude au Professorat d'Éducation Physique et Sportive). Leur expérience passée d'enseignement correspondait en général à la durée de deux mois de stage en établissement scolaire pour les années précédant le CAPEPS et aux trois premiers mois de leur année de stage pré-professionnel. Leur titularisation en tant que professeur a été obtenue à la fin de leur année de PLC2.

Recueil des données

Deux catégories de données ont été recueillies: (a) des données d'observation et d'enregistrement pendant l'action: l'enregistrement vidéo de l'action des enseignants et des élèves à l'aide d'une caméra VHS sur pied et d'un micro HF, (b) des données d'autoconfrontation a posteriori: l'auto-estimation à partir de l'enregistrement vidéo des enseignants du caractère plus ou moins confortable de leurs actions sur une échelle de "Confort - Inconfort" en huit points: de 1 (confortable) à 8 (très inconfortable), et l'enregistrement audio des verbalisations lors d'un entretien d'autoconfrontation des enseignants au sujet de leur leçon.

Cet entretien d'autoconfrontation s'est déroulé immédiatement après la leçon. Il a consisté à soumettre les enseignants à l'enregistrement vidéo de leur leçon, et leur demander de décrire ce qu'ils faisaient, ce qu'ils pensaient, ce qu'ils prenaient en compte pour agir, ce qu'ils percevaient ou ressentaient. Lors de l'autoconfrontation, les enseignants devaient estimer sur une échelle graduée le confort perçu sans justifier, argumenter ou généraliser cette estimation. Ils pouvaient à tout moment modifier les valeurs estimées ou évoquer ce qu'ils ressentaient au fil de la séquence vidéo.

Traitement des données

L'unité élémentaire du cours d'expérience (U) est caractérisée par un verbe d'action suivi d'un complément d'objet direct ou indirect. suivant:

L'identification des autres composantes des signes hexadiques a été réalisée grâce au questionnement

Pour le représentamen (R): quel(s) élément(s) rappelé(s), perçu(s) de la situation fait (font) signe(s) pour l'enseignant à l'instant étudié ?

Pour les préoccupations (eR): quelles sont les préoccupations saillantes chez l'enseignant en fonction de ce qui fait signe pour lui dans la situation ?

Pour l'actualité potentielle $(\mathrm{aR})$ : quelles sont les attentes de l'enseignant à l'instant étudié, prolongeant concrètement ses préoccupations $(\mathrm{eR})$ ?

Pour le référentiel (sR): quelles sont les connaissances mobilisées par l'enseignant à l'instant étudié ?

Pour l'interprétant (I): quelles connaissances sont en cours de construction selon un processus de confirmation ou d'infirmation?

Analyse locale du cours d'expérience de Gaëlle

\section{Résultats}

Nous présentons ici un extrait de ce programme de recherche: l'analyse locale de l'expérience de Gaëlle enseignante débutante en Éducation Physique, observée avec une classe de première de 26 élèves, au cours d'une

\footnotetext{
${ }^{4}$ En procédant à la description et à l'interprétation de quelques minutes d'un seul cours d'expérience, nous souhaitons mettre en évidence la fécondité de cette méthode pour analyser à un grain fin l'expérience en situation professionnelle.
} 
leçon de Volley ball. Les élèves participaient sans résistance ni opposition au cours. La leçon observée était la quatrième sur huit d'un cycle de Volley ball.

Le cours d'expérience étudié se situe de la $10^{\text {ème }}$ à la $13^{\text {ème }}$ minute de la leçon. Il débute lorsque Gaëlle attend les élèves lors de l'échauffement pour leur annoncer le commencement des étirements. Elle appelle les élèves et leur dit de commencer les exercices d'étirement: "Allez, c'est parti, les jambes... On s'étire ! ...". Sa préoccupation est de leur annoncer le début des étirements sans consignes spécifiques de sa part pour voir ce qu'ils savent faire. Elle attend de leur part qu'ils s'étirent seuls en reprenant les exercices déjà réalisés lors des leçons précédentes en Volley ball et lors du cycle d'Athlétisme. Les élèves s'étirent assis par terre; Gaëlle observe leur action en se déplaçant entre eux (Signe 1).

\section{Signe Hexadique 1}

Gaëlle - Volley ball ( $3^{\text {ème }}$ leçon) - Classe de $1^{\text {ère }}$ STT (26 élèves)

\section{Comportements en classe}

$11^{\prime} 12 "$

L'enseignante pose un ballon de Volley ball par terre et annonce le début des étirements en ayant une posture fléchie vers les élèves. Les élèves s'assoient en s'éloignant les uns par rapport aux autres et commencent à s'étirer.

\section{Communications en classe}

Enseignante: "Allez, c’est parti, les jambes...On s'étire... Étirements... Étirements ! On en profite... On souffle, on récupère...".

\section{Extraits d'autoconfrontation}

Enseignante: "Je dis par terre, $\mathrm{j}$ 'aime bien les mettre tout le temps devant moi ... Je ne leur dis rien exprès [Pas de consignes concernant les étirements] parce qu'on a déjà fait depuis le début des étirements comme ça... Aussi en athlé...[Lors du cycle précédent d'Athlétisme]. Pour voir un petit peu ce qu'ils font...".

Enseignante: "C'est plutôt calme, c'est un moment agréable [Les étirements] même s'il y en a qui le font plus ou moins [Les étirements], je les presse moins en fait... Je passe dans le groupe, je regarde ce qu'ils font, je supervise leur activité".

\section{CONSTRUCTION LOCALE}

SIGNE 1

\section{"Confort-Inconfort": 2/8}

\section{Représentamen (R)}

- Les élèves calmes et assis par terre en train de s'étirer

- Les étirements des élèves lors des leçons précédentes (notamment lors du cycle d'Athlétisme)

\section{$\sum$ Préoccupation(s) (eR)}

- Superviser les étirements des élèves en se déplaçant entre eux

\section{$\sum$ Actualité potentielle (aR)}

- Attentes liées à l'étirement des élèves seuls et plus particulièrement à leur reprise des étirements réalisés lors des leçons précédentes

\section{$\sum$ Référentiel (sR)}

- Attentes liées à l'étirement des élèves sans exigence technique particulière

- La position assise des élèves permet de superviser leur action plus facilement

- Ne pas donner de consignes initiales permet d'apprécier ce que les élèves savent faire sans aide de l'enseignant

- Les élèves savent s'étirer seuls 
Sentiment: Confort "d'un moment agréable"

Unité élémentaire (U)

- Observe l'action des élèves en se déplaçant entre eux

\begin{abstract}
Interprétant (I)
- Confirmation de la fiabilité du type: "Les étirements constituent une situation agréable d'enseignement pendant laquelle les élèves sont calmes et assis"
\end{abstract}

L'observation des élèves calmes et assis par terre en train de s'étirer confirme à Gaëlle que les étirements constituent un "moment agréable". Elle indique la valeur de 2/8 sur l'échelle de "Confort - Inconfort". Ses attentes vis-à-vis des élèves concernent davantage leur participation d'ensemble aux étirements que des exigences techniques particulières à la façon de s'étirer: "Je les presse moins, même s'il y en a qui font plus ou moins l'exercice demandé".

Un élève (Alexis) s'exclame: "Ah ! ça tire là..." Elle se retourne et se dirige vers lui rapidement (Signe 2) l'observe les mains sur les hanches, lui dit: "Oui... Allez ... Sérieusement... Qu'est-ce que tu étires là ? Dis-moi ...".

La rencontre avec Alexis s'inscrit dans l'histoire d'une relation particulière avec un élève "pas toujours bien dans son assiette". Ce qui fait signe pour l'enseignante dans la situation présente est ancré sur trois indices: (a) la remarque d'Alexis dans son dos (Alexis l'interpelle, elle se retourne), (b) la position assise d'Alexis jugée comme le signe d'un engagement insuffisant dans l'action demandée par l'enseignante: "je suis venue parce qu'il était en position "plage" un peu...", (c) la mémoire des moments difficiles déjà vécus avec Alexis: "C'est un élève qui a vite fait de décrocher".

Gaëlle effectue en marchant un tour et demi autour d'Alexis tout en l'observant. Alexis la fixe du regard. Elle lui dit: "Ne donne pas d'à-coups, fais-le sérieusement... Reste le dos droit, pas d'à-coups...". Elle l'aide ensuite à exécuter ses étirements en poussant son dos avec une main, puis lui dit: "... À force, ça ira loin si tu fais ça tous les jours comme ça...".

La description de cette interaction met en évidence: (a) un changement dans la proximité de l'enseignante vis-à-vis de l'élève: elle effectue un déplacement, les mains sur les hanches, d'un tour et demi autour de lui en conservant une distance constante vis-à-vis de lui, suivi d'un rapprochement et d'un contact corporel pour corriger sa façon de s'étirer, (b) un changement dans la tonalité de ses prises de parole: Elle adopte au début de l'interaction une intonation sèche et directive, suivie d'une intonation plus conviviale et incitative: "Bon et bien tu restes comme ça au lieu de faire des allers et retours (des à-coups dans l'étirement)...".

Le changement de mode d'intervention traduit l'évolution de ses préoccupations vis-à-vis de cet élève: dans un premier temps sa préoccupation est de contrôler Alexis dont elle se méfie. Elle veut lui montrer son désaccord vis-à-vis de sa position assise qu'elle estime non conforme à ses attentes. Elle sait qu'il a vite fait de décrocher et que le fait de lui montrer sa présence est un moyen de le contrôler. Puis sa préoccupation dans un deuxième temps évolue vers l'entretien d'un dialogue avec Alexis. Elle sait aussi qu'Alexis est un élève avec qui il faut souvent discuter et que le dialogue avec ce dernier est préférable à une intervention directive.

Ces connaissances sont relatives à cet élève particulier et aux modes d'intervention qui se sont révélés efficaces par le passé avec lui: le contrôler par une présence physique et établir avec lui un dialogue. Elles délimitent et déterminent son action. Cette action est efficace, ce qui est en partie à l'origine du caractère confortable évoqué par l'enseignante (2/8 sur l'échelle de "Confort - Inconfort").

Après avoir bouclé un deuxième tour autour de lui, elle rejoint le bord du gymnase. Elle revient face à la classe en estimant pouvoir gagner du temps en délivrant aux élèves des consignes en même temps qu'ils s'étirent (Signe 3).

Lors de la présentation de la suite de l'échauffement, elle hésite sur le nombre d'élèves dans chaque équipe. Un silence ponctue son intervention. Gaëlle montre l'exercice d'étirement suivant en levant les bras, parle de "petits travails" de manipulation de ballons. Une élève la reprend: "Des petits travaux !" (Rires de la classe). Gaëlle en sourit (silence) (Signe 4), hésite (silence) et finalement décide de différer la présentation des consignes du travail avec ballons (Signe 5) en disant aux élèves: "... On va faire ça d'abord [Les étirements des épaules] et je vous expliquerai la suite après...".

Lors de la faute de français, l'enseignante sourit en réponse au rire des élèves. Son action est à la fois constituée de sollicitations, de tâtonnements et d'ajustements face aux élèves. Ses hésitations semblent perçues par ces derniers et éveillent chez eux exclamations et expressions amusées. Elle indique un ressenti d'inconfort de 5/8 et

\footnotetext{
${ }^{5}$ Les signes hexadiques 2, 3,4, 5 et 6, permettant la description et l'interprétation de l'expérience de l'enseignante, ne sont pas présentés dans ce texte.
} 
évoque lors de l'autoconfrontation son hésitation, son manque de clarté. Elle reconnaît que son attitude souriante devant les élèves est davantage une façon de masquer son inconfort et de ne pas paraître perturbée devant les élèves.

Plusieurs événements sont observés lors de la présentation des consignes aux élèves: (a) les hésitations de l'enseignante quant au nombre de ballons et au nombre de joueurs par équipe, (b) sa faute de Français sur le pluriel de "travail" reprise spontanément par les élèves, (c) l'utilisation du futur puis du présent dans la même consigne: "Vous prendrez un ballon pour trois [...]. Vous prenez un petit espace, on commence comme ça...".

Gaëlle perçoit le manque de clarté de ses consignes: "... Mon explication n'est pas claire et précise du fait de mes hésitations...", et les signes de l'attention moins bonne des élèves: "Là ils parlent, je sens qu'ils n'écoutent pas". Elle décide de différer la délivrance des consignes du travail avec ballons: "Je voulais leur expliquer l'exercice qui allait suivre... Et là, j'abandonne...[...] Ça allait faire trop...". Sa décision correspond à l'abandon de ce qu'elle avait prévu de présenter initialement à la classe. Elle s'adapte dans l'urgence devant des élèves qui ne s'étirent plus et qui ne l'écoutent plus pour la plupart.

Gaëlle exprime un ressenti d'inconfort de 5/8 sur l'échelle de "Confort - Inconfort". Son inconfort émerge: (a) de ses silences, de ses hésitations sur le nombre de ballons, de sa faute de Français, (b) des exclamations, des rires, de l'amusement des élèves entraînant une dégradation de leur étirement et de leur écoute. Elle se sent mal à l'aise dans cette situation qui se complique pour elle: l'opportunité de gagner du temps en profitant des circonstances favorables liées au calme des élèves se transforme en une situation confuse et inconfortable de laquelle elle doit maintenant s'extraire.

Gaëlle dit aux élèves (12'41"): "... On va faire ça d'abord et je vous expliquerai la suite après...". La plupart des élèves se lèvent pour aller chercher les ballons sans poursuivre l'étirement des bras et des épaules. L'enseignante se déplace entre les élèves en montrant l'étirement prévu initialement et dit: "Les bras, les bras, les épaules, les épaules...". Certains élèves, près d'elle, l'imitent, mais la plupart se déplacent vers les ballons. L'enseignante réalise alors un déplacement circulaire en marchant entre les élèves. À ce moment, peu d'élèves la regardent. Elle marche environ deux mètres, le regard vers le sol. Elle relève la tête, croise Alexis ayant pris un ballon, qui s'arrête et dit: "Ah ? mais heu...". Elle le regarde en souriant et dit: "Allez, c'est parti..." (Signe 6).

Les élèves se déplacent rapidement pour prendre les ballons, tronquant les étirements des épaules souhaités par l'enseignante. Il y a incompréhension de la part des élèves qui pensent que les étirements des épaules sont terminés (pourtant Gaëlle insiste), ou tentative collective visant à précipiter le mouvement pour jouer plus vite avec les ballons. L'hésitation de Gaëlle lors de la présentation des consignes offre aux élèves la possibilité d'aller chercher les ballons et d'échapper à la fin des étirements des épaules.

L'enseignante insiste, verbalement et en levant les bras, pour maintenir les étirements des épaules. Ce qui fait signe pour elle dans la situation est ancré sur trois indices: (a) Alexis un ballon en main, (b) la moitié des élèves en déplacement vers les ballons, (c) l'étirement des épaules effectué par quelques élèves seulement.

$\mathrm{Au}$ moment où elle rencontre Alexis, elle accepte publiquement le déplacement des élèves vers les ballons en annonçant discrètement: "C'est parti". Cette décision constitue en moins de 15 secondes un renoncement supplémentaire par rapport à ce qu'elle avait prévu et un nouvel ajustement de son action à l'action non maîtrisée des élèves.

Lors de ce deuxième renoncement, l'enseignante procède en deux temps. Premièrement, les bras encore levés, elle décide de renoncer aux étirements des épaules. Elle l'annonce discrètement en croisant Alexis. Les bras encore levés témoignent de l'intention initiale de maintenir l'étirement des épaules. Deuxièmement, elle insiste, en baissant les bras, sur l'organisation rapide du travail avec les ballons. L'incitation est moins discrète.

Ses préoccupations sont alors de "Donner les indices d'un contrôle de l'action des élèves" le plus rapidement possible et de "Ne pas montrer le trouble relatif à la perte de contrôle des élèves". En effet, elle indique la valeur de 6/8 sur l'échelle de "Confort - Inconfort". Cet inconfort est lié à la perte du contrôle des élèves qui échappent à la fin des étirements.

Lors de cette perte de contrôle des élèves, les connaissances mobilisées sont: (a) les élèves préfèrent jouer au ballon que de s'étirer, (b) quand les élèves se précipitent sur les ballons, il est difficile d'intervenir, (c) les étirements ne constituent pas l'essentiel de cette leçon de Volley ball. Ces connaissances constituent des éléments d'interprétation des raisons possibles d'agir des élèves et d'évaluation des conséquences de la perte de contrôle de leur action. Leur mobilisation ne permet pas de contrôler les élèves et n'est pas efficace dans ces circonstances imprévues. Cette inefficacité contribue à l'inconfort ressenti.

Gaëlle revient près du bord du gymnase où les ballons sont rangés, baisse lentement les bras restés en extension (certaines filles sur son passage baissent les bras en même temps) et dit: "Allez... Rapidement !". Elle s'accroupit et compte à voix haute des ballons: "1, 2, 3 ...". 


\section{Discussion}

Les résultats de l'étude de ce cours d'expérience nous incitent à proposer une discussion en deux points relativement à l'engagement émotionnel de Gaëlle et aux petites erreurs pédagogiques contribuant à la dégradation de la situation d'enseignement de Gaëlle.

L'engagement émotionnel de Gaëlle

$\mathrm{Au}$ gré des circonstances, le vécu de l'enseignante révèle des îlots instables de confort et d'inconfort. En quelques secondes, son confort lié à "un moment agréable" pendant les étirements évolue vers des sensations plus désagréables lors des hésitations et de la perte anodine et sans conséquence du contrôle des élèves. Cette étude nous permet de mieux comprendre l'évolution très rapide et circonstancielle des ressentis plus inconfortables de Gaëlle. Deux aspects principaux sont à l'origine de cet inconfort: son indécision face aux élèves et l'ajustement de son action à celle non prévue et non maîtrisée des élèves.

Gaëlle perçoit lors de la présentation des consignes son manque de clarté, ses hésitations et ses silences. Son attitude détachée face aux élèves est une "une contrefaçon" pour masquer en souriant son trouble intérieur. Se laisser envahir par des émotions, les partager avec les élèves peut être vécu, pour un enseignant débutant, comme une remise en cause de son statut d'enseignant.

La perte de contrôle des élèves est à peine visible pour un observateur extérieur: l'enseignante baisse les bras au sens propre et figuré mais sans montrer le moindre signe de contestation ou de trouble. Pourtant, elle exprime son inconfort: "Là, ça n'est pas terrible !". Sa façon d'annoncer la mise en place de l'exercice suivant sans perdre de temps lui permet de minimiser cette perte de contrôle et d'estomper ce trouble en avançant dans l'action. Comme si le fait d'avancer lui permettait de quitter cette situation embarrassante et de reprendre en main les élèves. Dans ce sens, le "Rapidement" adressé à la classe est une tentative pour recréer le lien entre l'action des élèves et son ajustement tardif.

Dans cette mesure, les décisions en classe sont à comprendre au travers de l'évolution de la tonalité émotionnelle des actions de l'enseignante. Il ne s'agit pas de réduire l'analyse des émotions à une relation de causalité entre un ressenti inconfortable et une décision conséquente, mais de souligner la contribution de la tonalité émotionnelle lors des décisions de l'enseignante dans la classe. Dans notre étude, Gaëlle modifie ses plans lorsque l'inconfort s'installe, lorsqu'elle perçoit de façon désagréable la situation. Le ressenti de son engagement face aux élèves constitue alors l'essence même de ses décisions-en-action. Dès lors, ses décisions en classe sont contenues en puissance dans cette dynamique émotionnelle.

Que la tonalité émotionnelle soit confortable ou inconfortable, l'enseignante dans notre étude coupe court au déploiement d'émotions en classe en interrompant la coordination, en changeant ses plans ou en "plongeant dans une autre action". Elle tente de ne pas laisser trahir ses émotions ou en tout cas de ne pas en donner l'impression. Il s'agit pour elle de faire bonne figure, de donner une image d'elle-même consistante. Elle procède alors par "accords de surface" (Goffman, 1974) pour donner l'illusion d'une maîtrise des élèves. Ce jeu de pertinence conjoncturelle consiste à donner l'impression de contrôler les événements, quand bien même ces derniers lui échappent en partie, tout en dissimulant ses émotions.

Des petites erreurs pédagogiques contribuant à la dégradation de la situation d'enseignement de Gaëlle

Nous avons décrit l'action de l'enseignante en insistant sur des oublis, des hésitations, des renoncements et des ajustements. Une série de petites "erreurs pédagogiques" 6 a été commise par elle au cours de cet épisode: perte de vue de la classe au profit d'un élève seul; durée inadaptée de l'exercice; non-prévision du partage de la classe en équipes et du nombre de ballons par rapport au nombre d'élèves; délivrance de consignes trop nombreuses; sollicitation des élèves dans deux registres d'action simultanés; petite faute de Français; hésitations avec un changement de temps dans les consignes.

Ces erreurs peuvent dépasser le caractère singulier de cette étude. En effet, il nous semble que ces "tâtonnements pédagogiques" sont inhérents à l'inexpérience des enseignants débutants ${ }^{7}$ et raisonnablement généralisables.

\footnotetext{
${ }^{6} \mathrm{Si}$ nous faisons en sorte que le recueil des données ne constitue pas une gêne pour l'acteur (tout en admettant que notre présence et celle du dispositif d'observation dans la situation de travail transforment cette dernière), nous n'hésitons pas à nous engager a posteriori dans l'interprétation des données. Il ne s'agit plus seulement d'une description la plus neutre possible de l'expérience professionnelle mais d'une coopération entre un chercheur et des enseignants débutants. Selon le cadre ergologique de Schwartz (1997), cette coopération s'apparente à une action sociale de formation répondant à une double visée épistémique et transformative.

${ }^{7}$ Ces erreurs sont repérées dans d'autres cours d'expérience de notre programme de recherche.
} 
Lorsque Gaëlle revient devant les élèves après les conseils donnés à Alexis, elle estime qu'ils sont capables de faire deux choses en même temps: s'étirer tout en écoutant les consignes des exercices suivants. Cette estimation pourrait surprendre des enseignants plus chevronnés qui, par expérience, resteraient plus prudents quant à la capacité de travail en double tâche accordée ici aux élèves. En effet, ils savent qu'ils ne peuvent pas attendre des élèves simultanément la réalisation satisfaisante d'une action et l'écoute attentive de consignes pour une autre action. La dégradation progressive des étirements des élèves et de leur écoute contribue à diminuer la fiabilité de son estimation initiale.

D'autre part, si les élèves ne parviennent pas à faire deux choses à la fois (continuer de s'étirer et écouter les consignes de la suite de la leçon) Gaëlle n'y parvient pas non plus: elle ne parvient pas à maintenir leur étirement pendant qu'elle présente la suite de la leçon. Elle se focalise totalement sur sa présentation, hésite et perd peu à peu le contrôle de l'action des élèves qui se lèvent et se dirigent vers les ballons.

Plutôt que de se satisfaire de la période calme des étirements pendant laquelle elle aurait pu superviser l'action des élèves, elle anticipe, se projette dans sa leçon et cherche à gagner du temps. De ce fait elle ne voit plus l'action des élèves et contribue en grande partie à provoquer leur déplacement vers les ballons. Gaëlle se met ellemême en difficulté: la pression temporelle qu'elle subit est due au fait qu'elle veuille en priorité respecter son plan de leçon.

\section{Conclusion}

La comparaison systématique de différents cours d'expérience nous a permis de repérer des régularités au niveau de l'agir professionnel des enseignants débutants ${ }^{8}$. Ces derniers agissent et décident quotidiennement en fonction de la tonalité émotionnelle de leur expérience en classe, c'est-à-dire en fonction de leurs sentiments de confort ou d'inconfort ou de leurs souvenirs d'expériences vécues de façon plus ou moins confortable. Si les émotions des enseignants débutants constituent la base de l'intelligibilité adaptative de leur action, contenant en puissance leurs décisions en classe, le dévoilement et la compréhension en formation initiale de l'épaisseur émotionnelle de leur expérience professionnelle sont indispensables. Il s'agit de leur permettre individuellement ou collectivement d'exprimer leurs états affectifs comme la totalité synthétique de leur engagement en classe, leurs sentiments en fonction de ce qui leur a fait signe en classe et leurs émotions-types relatives au repérage de la régularité de ressentis confortables ou inconfortables dans des situations d'enseignement relativement similaires.

\section{Références}

Damasio, A.R. (1999). Le sentiment même de soi. Corps, émotions, conscience. Paris: Edition Odile Jacob.

Durand, M. (1998). Teaching action in physical education. Cagigal Lecture at the AIESEP World Conference, Adelphi, July.

Durand, M. (2000). Chronomètre et survêtement: reflets de l'expérience quotidienne d'enseignants d'Éducation Physique. Paris: Revue EPS.

Goffman, E. (1974). Les rites d'interaction. Paris: Editions Minuit.

Peirce, C.S. (1978). Écrits sur le signe. Paris: Editions du Seuil.

Ria, L. (2001). Les préoccupations des enseignants débutants en Education Physique et Sportive. Etude de l'expérience professionnelle et conception d'aides à la formation. Thèse de doctorat STAPS non publiée. Université de Montpellier 1.

Ria, L., Saury, J., Sève, C., Durand, M. (2001). Les dilemmes des enseignants débutants: Etudes lors des premières expériences de classe en Education Physique. Science et Motricité, 42, 47-58.

Schwartz, Y. (Ed.) (1997). Reconnaissance du travail. Pour une approche ergologique. Paris: PUF.

Theureau, J. (1992). Le cours d'action: Analyse sémio-logique. Essai d'une anthropologie cognitive située. Berne: Peter Lang.

Theureau, J. (2000). Anthropologie cognitive et analyse des compétences. In J.M. Barbier (Ed.), L'analyse de la singularité de l'action (pp.171-211). Paris: PUF.

\footnotetext{
${ }^{8}$ La comparaison s'effectue à partir de l'organisation globale du cours d'expérience de plusieurs enseignants: c'est-àdire à partir de l'organisation de structures significatives plus larges. Par exemple, l'organisation des séquences constituées de plusieurs unités élémentaires autour d'une même préoccupation. Les séquences-types de leurs cours d'expérience rendant compte de leurs préoccupations-types (Ria, 2001).
} 